\title{
Development and application of the near-infrared and white-light thoracoscope system for minimally invasive lung cancer surgery
}

\author{
Yamin Mao \\ Kun Wang \\ Kunshan He \\ Jinzuo Ye \\ Fan Yang \\ Jian Zhou \\ $\mathrm{Hao} \mathrm{Li}$ \\ Xiuyuan Chen \\ Jun Wang \\ Chongwei Chi \\ Jie Tian
}




\title{
Development and application of the near-infrared and white-light thoracoscope system for minimally invasive lung cancer surgery
}

\author{
Yamin Mao, ${ }^{a, b, c, \dagger}$ Kun Wang, ${ }^{a, b, \dagger}$ Kunshan He, ${ }^{a, b, c}$ Jinzuo Ye, ${ }^{a, b, c}$ Fan Yang, ${ }^{d}$ Jian Zhou, ${ }^{d}$ Hao Li, ${ }^{d}$ Xiuyuan Chen, ${ }^{d}$ \\ Jun Wang, ${ }^{d}$ Chongwei Chi, ${ }^{a, b, *}$ and Jie Tian ${ }^{\mathrm{a}, \mathrm{b}, *}$ \\ ${ }^{a}$ Chinese Academy of Sciences, Institute of Automation, Key Laboratory of Molecular Imaging, Beijing, China \\ ${ }^{b}$ Beijing Key Laboratory of Molecular Imaging, Beijing, China \\ 'University of Chinese Academy of Sciences, Beijing, China \\ dPeking University People's Hospital, Department of Thoracic Surgery, Beijing, China
}

\begin{abstract}
In minimally invasive surgery, the white-light thoracoscope as a standard imaging tool is facing challenges of the low contrast between important anatomical or pathological regions and surrounding tissues. Recently, the near-infrared (NIR) fluorescence imaging shows superior advantages over the conventional white-light observation, which inspires researchers to develop imaging systems to improve overall outcomes of endoscopic imaging. We developed an NIR and white-light dual-channel thoracoscope system, which achieved high-fluorescent signal acquisition efficiency and the simultaneously optimal visualization of the NIR and color dual-channel signals. The system was designed to have fast and accurate image registration and high signal-to-background ratio by optimizing both software algorithms and optical hardware components for better performance in the NIR spectrum band. The system evaluation demonstrated that the minimally detectable concentration of indocyanine green (ICG) was $0.01 \mu \mathrm{M}$, and the spatial resolution was $35 \mu \mathrm{m}$. The in vivo feasibility of our system was verified by the preclinical experiments using six porcine models with the intravenous injection of ICG. Furthermore, the system was successfully applied for guiding the minimally invasive segmentectomy in three lung cancer patients, which revealed that our system held great promise for the clinical translation in lung cancer surgeries. () 2017 Society of Photo-Optical Instrumentation Engineers (SPIE) [DOI: 10.1117/1.JBO.22.6.066002]
\end{abstract}

Keywords: fluorescence image-guided surgery; minimally invasive surgery; thoracoscope; indocyanine green; lung cancer surgery; thoracic duct; pulmonary segments.

Paper 160679RR received Oct. 6, 2016; accepted for publication May 11, 2017; published online Jun. 5, 2017.

\section{Introduction}

Lung cancer remains the leading cause of cancer-related death worldwide, resulting in 1.4 to 1.6 million deaths annually. ${ }^{1-3}$ Because of a better postoperative recovery versus open surgery, minimally invasive surgery (MIS) was the prior choice of surgical interventions. ${ }^{4}$ However, current MIS highly relies on the intraoperative color imaging guidance with conventional thoracoscopy system. Although the white-light system plays a crucial role in MIS, surgeons still have to face the challenge of discriminating important anatomical and pathological structures from their surrounding tissues, because frequently, they both have similar morphology, texture, and color in white-light images. ${ }^{5-9}$ Therefore, a new imaging technology with better ability of identifying the region of interests is desired for guiding MIS.

In recent years, thoracoscopy incorporating advanced nearinfrared (NIR) fluorescence imaging technologies has the potential to provide highly sensitive and specific imaging guidance of surgically interested regions. ${ }^{10-12}$ Different from the color imaging, NIR fluorescence imaging only illuminates certain regions inside the field-of-view (FoV) and provides pathological information several millimeters beneath the tissue surface, which results in many advantages in the intraoperative usage. ${ }^{13}$ Some

*Address all correspondence to: Chongwei Chi, E-mail: chongwei.chi@ia.ac. cn; Jie Tian, E-mail: jie.tian@ia.ac.cn

†These authors contributed equally to this work. recent preclinical and clinical applications already proved the feasibility and benefits of NIR image-guided MIS, such as its application in colorectal surgery, ${ }^{14-17}$ bladder cancer, ${ }^{18,19}$ cholecystectomy,${ }^{20}$ liver cancer ${ }^{21}$ and gastric cancer. ${ }^{22}$

Furthermore, NIR fluorescence imaging also showed its potential in minimally invasive lung cancer surgery (MILCS). ${ }^{13,23-25}$ Vivek et al. successfully detected lung sentinel lymph node with a self-developed fluorescence endoscopic system. ${ }^{13}$ However, the optimization of the endoscopic NIR imaging system in sensitivity and ergonomic clinic design for improving MILCS in clinical practices remains extremely underexplored. For example, in lung cancer surgery, segmentectomy is commonly considered to be a better choice than lobectomy for achieving a complete resection as well as preserving more lung function, ${ }^{26,27}$ but it is still a clinical challenge for surgeons to find clear intersegmental planes during MILCS. Moreover, the iatrogenic injury of thoracic duct may cause chylothorax and reoperation of a patient in MILCS, whereas it is hard to distinguish the thoracic duct from its surrounding tissues with current white-light thoracoscope $^{28,29}$ Thus, the endoscopic NIR imaging technology may be a new choice for solving all these clinical issues in MILCS.

In this study, we designed a two-camera system for the challenge of satisfying the optimal visualization of color imaging, the maximization of NIR light collection efficiency, and ergonomic clinic design. Aiming for the clinical translation, this fused NIR

1083-3668/2017/\$25.00 ๑ 2017 SPIE 
and white-light thoracoscope (FFWT) system was specially designed for imaging indocyanine green (ICG) (excitation: $778 \mathrm{~nm}$ and emission: $830 \mathrm{~nm}$ ), since it is the only FDA approved NIR dye. After a series of evaluation, the FFWT system demonstrated very high NIR collection efficiency in the emission wavelength of ICG. Its minimal detection concentration of ICG was about 10 times higher than the published system for MILCS. ${ }^{13}$ It also achieved quick and robust image registration because of its unique design of the mechanical structure and optical pathway. With the help of ICG, our system successfully identified intersegment planes and thoracic ducts in six porcine models. Furthermore, we recruited three lung cancer patients to verify the performance of the FFWT system in image-guided MILCS. The preliminary results proved that it can effectively identify intersegment planes with signal-to-background ratio (SBR) of $21.5 \pm 0.3 \mathrm{~dB}$. All these experiments revealed that the newly developed FFWT system holds great promise for helping surgeons to achieve more accurate lung segment resection and avoid iatrogenic injuries in MILCS.

\section{Materials and Methods}

First, to achieve simultaneous color and NIR dual-channel thoracoscopic imaging, we designed the imaging components of FFWT (Fig. 1) based on the following four aspects.

(a)

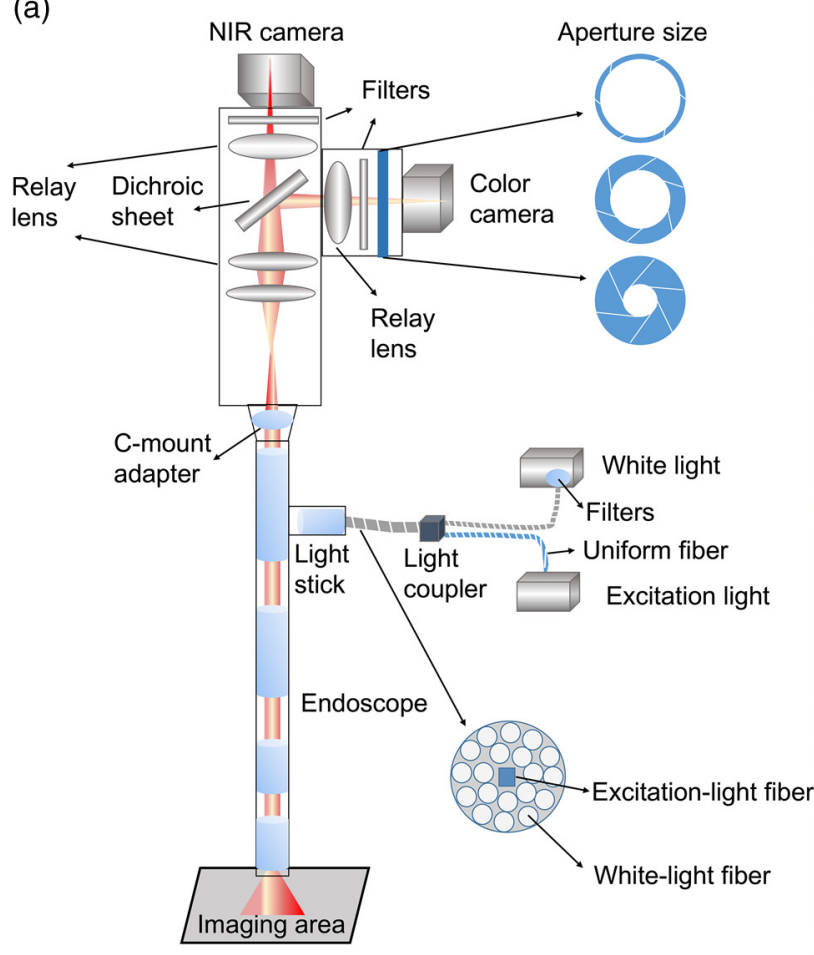

1. Optical path design: A clinically approved rigid thoracoscope optimized for capturing both visible and NIR light was specially selected to fit in the system (HOPKINS II 26003AGA, Karl Storz, Tuttlingen, Germany). For simultaneously acquiring color and NIR images, we specially developed an optical path to achieve the optimal performance for both color imaging (400 to $650 \mathrm{~nm}$ ) and ICG (excitation: $778 \mathrm{~nm}$, and emission: $830 \mathrm{~nm}$ ) imaging, since ICG was the only FDA approved NIR dye. Meanwhile, we presented a solution to achieve real-time image registration by designing the optical path for the same FoV of color and NIR cameras and applying quick robust algorithms for color and NIR images registration. Furthermore, the components' size and weight were optimized to satisfy real-time ergonomic clinical application and long-time handheld use.

2. Imaging acquisition: To ensure high sensitivity, small size, and light weight of imaging camera, we, cooperating with Microview Inc., developed a light-weight science complementary metal oxide semiconductor (SCMOS) NIR camera (weight: 250 g, HK-A5100GM17, Microview, Beijing, China). Meanwhile, to

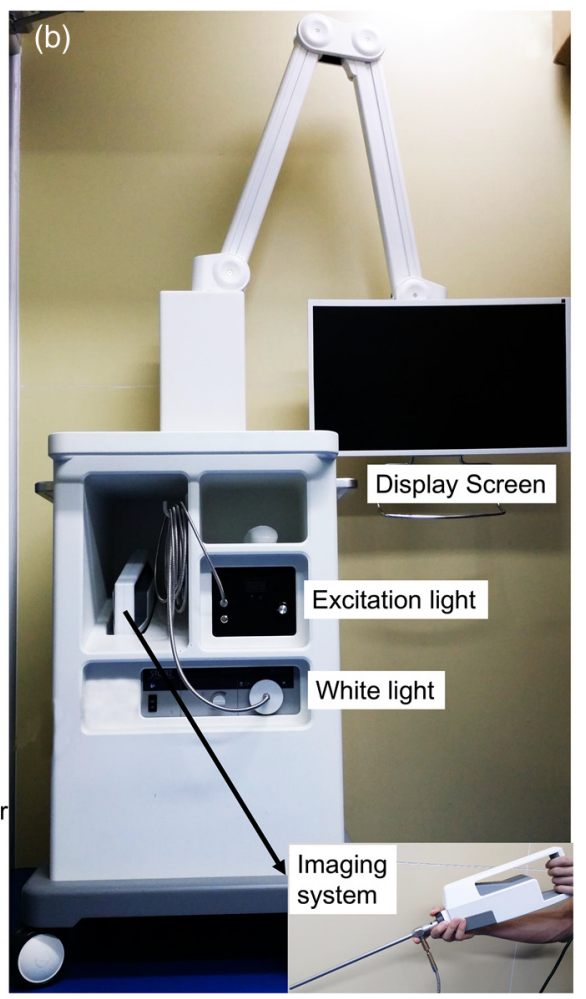

Fig. 1 The design of the imaging component and a picture of the FFWT system. (a) The schematic illustration of the imaging system. The NIR excitation light source and white-light source for illumination were coupled into thoracoscope fiber port through a multimode bifurcated fiber bundle. The fiber assignment was shown in the cross-sectional view, where the blue square represents excitation-light fiber, and white circles represent white-light fibers. Photons were collected from the thoracoscope and divided by a dichroic sheet. Light wavebands between 810 to $870 \mathrm{~nm}$ and 400 to $650 \mathrm{~nm}$ were separated by the dichroic sheet and captured by the NIR and color cameras, respectively. The aperture size of the color imaging was controlled as shown at the top-right corner of (a). (b) The picture of the entire FFWT system. The picture on the bottom right corner shows that the imaging system can be handheld with the ergonomically-designed handle. The display screen was connected with a mechanical arm with multidegree of freedom. The handheld component, light sources, screen, and control station were all installed in a trolley, so the FFWT system can be easily moved from one operation room to another. 
acquire color/NIR images with the same FoV, we customized a color camera (HK-A5100-GC17, Microview, Beijing, China) with the identical cell size of the NIR camera.

3. Illumination design: To provide simultaneous white and NIR excitation light and reduce light coupling loss, we developed a custom multimode bifurcated fiber bundle (Banglei Optoelectronic Technology Co. Ltd., Guangdong, China) to connect the white-light and excitation light source. Meanwhile, we specially developed a light stick and a uniform fiber to expand the facula area of NIR excited light, because the excited light was a point source.

4. Software development: In order to display color, NIR, and fused images in video rate, the software was developed with parameters of automatic adjustment function, image-processing algorithms, and quick imageregistration algorithms.

\subsection{Optical Path Design}

A self-developed light splitter was used for simultaneously acquiring color and NIR images. This module comprised a dichroic sheet (DMLP650, Thorlabs Ltd., Newton, New Jersey) and a set of relay lens. The dichroic sheet was used to divide light into two beams. One light beam with the wavelength between 810 and $870 \mathrm{~nm}$ was transmitted to the NIR camera. The other light beam with the wavelength between 400 and $650 \mathrm{~nm}$ was reflected to the color camera. To ensure the same FoV of color and NIR cameras, an adjustable structure was designed for mounting the dichroic sheet and reducing the mechanical error. Furthermore, the relay lens consisted of three sets of lens and filters, which were assembled for focusing the collected light on the camera sensor. Because the light transmission rates of the selected dichroic sheet and aspheric lens reached more than $95 \%$ in each favorable wavelength band (Fig. 2), the aperture size design ensured sufficient depth-ofview, reduced stray light, and increased brightness contrast of color imaging, and the whole light splitter was assembled in a very small cylindrical chamber (internal diameter: $2 \mathrm{~cm}$ and internal length: $4 \mathrm{~cm}$ ), the loss of the optical signal caused by light-splitting was minimized.

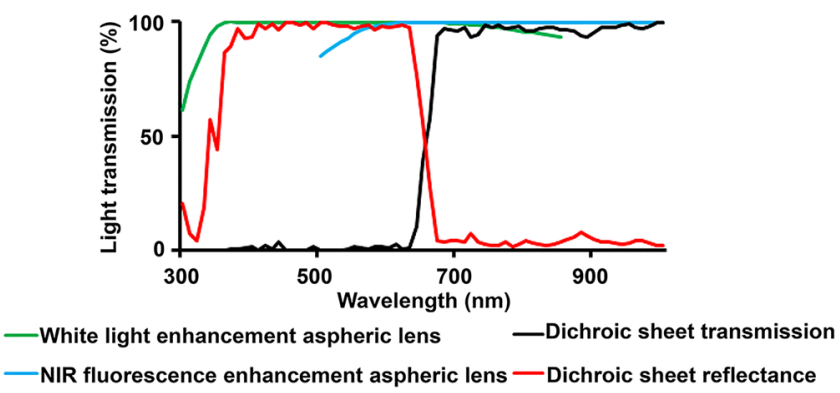

Fig. 2 The light transmission rate of utilized lens in the light-splitting module. The green and blue lines separately showed the light transmission of white-light and NIR-light enhancement aspheric lens. Both wavelengths (wavelength: 300 to $850 \mathrm{~nm}$ and 810 to $870 \mathrm{~nm}$ ) reached above $99 \%$. The black and red lines, respectively, represent the transmission efficiency and reflection efficiency of the dichroic sheet. Both reached above $95 \%$.
Besides that, the light-splitting module was coupled with a 0deg direction of view, 70-deg visual field, 10-mm diameter, and 31-cm length thoracoscope. We compared several commercially available rigid thoracoscopes provided by different manufacturers (Karl Storz, Olympus, and Stryker), and they were all clinically approved. Finally, the one with the best performance in the NIR spectrum was selected for experiments.

\subsection{Imaging Acquisition}

For the thoracoscopic imaging system design, it is difficult to choose a high-sensitivity, light-weight, and small-size camera. To balance the weight/size and sensitivity, we cooperated with Microview Inc. and developed a light-weight SCMOS NIR camera (weight: 250 g, HK-A5100-GM17, Microview, Beijing, China), which meets the requirement of sensitivity. Meanwhile, because NIR and color cameras were designed to have exactly the same cell size and pixel number (Table 1) and combined with the dichroic beam splitter, the dual-channel imaging inherently had the same FoV. This hardware feature ensured fast and accurate NIR and color imaging registration. Furthermore, the SCMOS NIR camera with high NIR-light imaging sensitivity facilitated weak fluorescent signal collection within 50-ms exposure time at each frame. Besides all these features, both cameras were very compact and light in weight (Table 1). To reduce the signal noise during acquisition, two filters (wavelength: $400-650 \mathrm{~nm}$ and $810-870 \mathrm{~nm}$ ) were fixed in front of two cameras, respectively [Fig. 1(a)]. The acquired raw data were transmitted to the computer in the control station through

Table 1 Key parameters of the newly developed FFWT system.

\begin{tabular}{|c|c|c|}
\hline Category & Specification & Description \\
\hline \multirow[t]{4}{*}{ NIR camera } & Resolution & $2560 \times 2160$ pixels \\
\hline & Cell size & $6.5 \mu \mathrm{m} \times 6.5 \mu \mathrm{m}$ \\
\hline & Size & $5.6 \times 6 \times 6 \mathrm{~cm}^{3}$ \\
\hline & Weight & $250 \mathrm{~g}$ \\
\hline \multirow[t]{4}{*}{ Color camera } & Resolution & $2560 \times 2160$ pixels \\
\hline & Cell size & $6.5 \mu \mathrm{m} \times 6.5 \mu \mathrm{m}$ \\
\hline & Size & $5.6 \times 6 \times 6 \mathrm{~cm}^{3}$ \\
\hline & Weight & $250 \mathrm{~g}$ \\
\hline \multirow{2}{*}{$\begin{array}{l}\text { Handheld imaging system } \\
\text { with the thoracoscope }\end{array}$} & Length & $58 \mathrm{~cm}$ \\
\hline & Weight & $1.15 \mathrm{~kg}$ \\
\hline \multirow[t]{3}{*}{ NIR excitation light } & Type & $\begin{array}{l}\text { SMA coupled } \\
\text { laser diode }\end{array}$ \\
\hline & Wavelength & $785 \mathrm{~nm}$ \\
\hline & Power & 0 to $5 \mathrm{~W}$ \\
\hline \multirow[t]{3}{*}{ White light } & Type & Light-emitting diode \\
\hline & Wavelength & 400 to $650 \mathrm{~nm}$ \\
\hline & Power & $300 \mathrm{~W}$ \\
\hline
\end{tabular}


the gigabit-per-second wires, so that the requirement of highspeed data transfer was guaranteed. As a result, the system could achieve real-time high-SBR dual-channel imaging with the frame rate above $20 \mathrm{fps}$.

\subsection{Illumination Design}

To simultaneously acquire color and NIR images, the NIR excitation light and white light were coupled into the thoracoscope. The NIR excitation light source (MW-SGX-785, Changchun Lei Shi Optoelectronic Technology Co. Ltd, Changchun, Jilin, China) used a 785-nm laser with continuously adjustable power from 0 to $5 \mathrm{~W}$. The white-light illumination was provided by a 300-W LED light source (Kenon Nova 300, Karl Storz, Tuttlingen, Germany) in the visible light window. A customized 3-m long multimode bifurcated fiber bundle was used to couple the NIR and white-light sources. It was $4.8 \mathrm{~mm}$ in diameter, and a NIR excitation-light fiber ( $400 \mu \mathrm{m}$ in diameter) was in the center of the bundle surrounded by multiple white-light fibers (Fig. 1). This multimode bifurcated fiber bundle was connected to the thoracoscope using standard endoscopic light source coupling.

\subsection{Software Development}

In order to realize the real-time intraoperative image registration, storage, and display, a customized software was developed. The image processing pipeline was shown in Fig. 3. The parameters controlled by our software includes exposure time, light intensity gain, and image binning for acquiring high-contrast dynamic images. All data collected from color and NIR cameras were transmitted into the computer through standard gigabitper-second wires. Benefited from the system structure design, color and NIR images have the same FoV and pixel number. As a result, the dual-channel imaging registration was easy, quick, and accurate without using a complicated coregistration algorithm. Furthermore, the entire interface of the software can be upgraded and extended, so other algorithms can be also added into the software.

\subsection{System Characteristics}

\subsubsection{Optical characteristics}

The spatial resolution of FFWT was measured by imaging a target board (USAF 1951, Thorlabs Ltd.) at the distance of 25, 50, and $75 \mathrm{~mm}$. Images were analyzed by finding whether the vertical stripes lost the contrast with adjacent lines. The same method was used to analyze the horizontal resolution. After these, the spatial resolution limit of the system was determined.

\subsubsection{Sensitivity characteristics}

The sensitivity characteristics of FFWT were tested by using a series of ICG (Yichuang Pharmaceutical Limited Liability Company, Dandong, China) solutions (1, 0.1, $0.01 \mu \mathrm{M})$. Meanwhile, for testing the background noise, the images of the phosphate buffered saline (PBS) solution at the same conditions were also obtained as a reference. The ICG and PBS solutions were placed in four 1.5-ml eppendorf (EP) tubes and imaged at 100ms exposure time. To avoid the disturbance between high dose solution and low dose solution, we separately measured each ICG solution alone. Then, all images were analyzed by an image processing software (ImageJ, National Institutes of Health, USA). We delineated the region of interest (RoI) on the tips of EP tubes with ICG and PBS solutions and calculated the mean fluorescence intensity of each RoI using ImageJ software. Then, the SBR in $\mathrm{dB}$ was calculated in Eq. (1):

$$
\mathrm{SBR}=20 \log \frac{I_{\mathrm{ICG}}}{I_{\mathrm{PBS}}},
$$

where $I_{\text {ICG }}$ denotes the mean fluorescence intensity of the ICG solution and $I_{\mathrm{PBS}}$ denotes the mean fluorescence intensity of PBS.

\subsection{In Vivo Preclinical Experiments in Porcine Models with FFWT}

The feasibility of FFWT was validated through in vivo animal experiments. The identification of the thoracic duct $(n=3)$ and intersegmental plane $(n=3)$ was performed in six 3- to 5month-old miniature porcine models ( 30 to $35 \mathrm{~kg}$ ). All experiments were approved by the Johnson \& Johnson Beijing Medical Science Centers. Before surgery, the pigs were anesthetized with $2 \%$ isofluorane and monitored by a physiological monitor. Pulmonary segmentectomy experiments were performed using 12-mg ICG with a concentration of $5 \mathrm{mg} / \mathrm{ml}$ through marginal ear vein injection. FFWT was used to image the pulmonary segment during the surgery. Real-time color, NIR, and fused images of the intersegmental plane were displayed on the screen and recorded as video data. After that, thoracic duct experiments were performed by injecting $1 \mathrm{mg}$ ICG subcutaneously at a concentration of $5 \mathrm{mg} / \mathrm{ml}$ into the

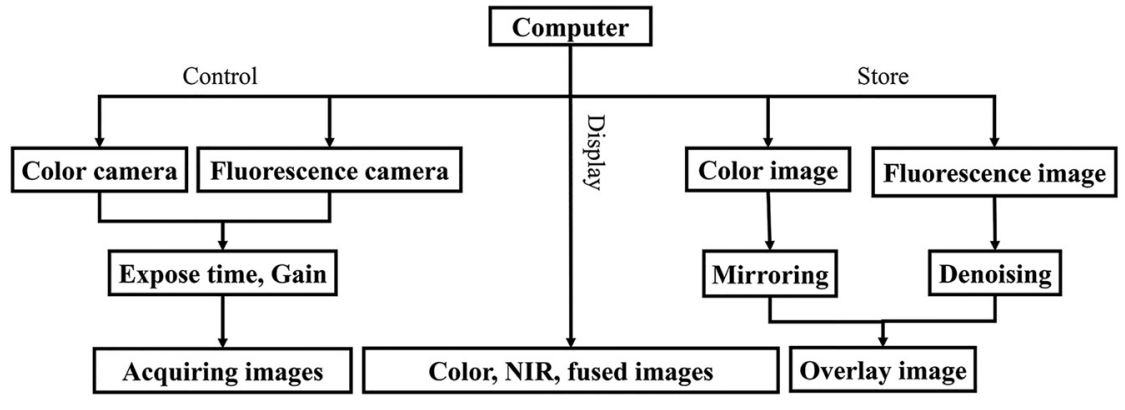

Fig. 3 The software pipeline of the FFWT system. The software provided the function of controlling expose time, gain, and mirroring of cameras. The color and fluorescence images were stored and fused, and then the color, fluorescence and fusing images were displayed for intraoperative identifying important anatomical or pathological features in real time. 



Fig. 4 System characteristics evaluation. (a) A USAF 1951 test target color image collected by the FFWT system at a working distance of $25 \mathrm{~mm}$. (b) The local enlarged image of (a). The three to six stripes did not lose the contrast with adjacent lines in vertical and horizontal directions (red outline). The corresponding spatial resolution of three to six stripes was $35 \mu \mathrm{m}$. (c) and (d) The optical resolution of FFWT was separately 63 and $79 \mu \mathrm{m}$ at the working distance of 50 and $75 \mathrm{~mm}$. (e) The fused images of different ICG solutions (1, 0.1 , and $0.01 \mu \mathrm{M})$ and one PBS solution at the exposure time of $100 \mathrm{~ms}$. (f) The SBR bar chart of different ICG solutions.

right leg, and the thoracic duct imaging was performed around 13 min later.

\subsection{In Vivo Clinical Experiments in Lung Cancer Patients with FFWT}

To evaluate the feasibility of the FFWT system in NIR imageguided segmentectomy, we recruited three patients previously scheduled for white-light thoracoscopic surgery. All patients signed the informed consent. This study was approved by the institutional ethics committee of Peking University People's Hospital (2015PHB157-01) and registered at ClinicalTrials. gov (NCT02611245). The study's inclusion criteria were as follows: (i) existing pulmonary nodules and prepared for thoracic surgery, (ii) no history of allergy to ICG, and (iii) aged between 18 and 75 years. The exclusion criteria were as follows: (i) preoperative liver dysfunction, (ii) known allergy to ICG, and (iii) with other uncontrollable complications. First, patients were intravenously injected with $0.4 \mathrm{mg} / \mathrm{kg}$ ICG after ligating the segmental pulmonary arteries. Then, we performed NIR imageguided segmentectomy with FFWT (Fig. 6). The surgical procedure was recorded in video rate. For each patient, we calculated the SBR of selected frames in each video.

\section{Results}

\subsection{System Parameters}

The parameters of FFWT were shown in Table 1. NIR camera and color camera have high resolution for acquiring high-quality images and the same cell size for imaging identical FoV. Moreover, two cameras with light weight and small size ensured user friendly operation.

\subsection{Optical Resolution}

We measured the optical resolution of FFWT at different distances $(25,50$, and $75 \mathrm{~mm})$, which were shown in Figs. 4(a)-4(d). The red outline part of Fig. 4(a) was magnified and placed in Fig. 4(b), which shows that the group number three, element six stripe did not lose the contrast with adjacent lines in vertical and horizontal directions. Therefore, the system's vertical and horizontal resolution was determined to be $35 \mu \mathrm{m}$ at a working distance of $25 \mathrm{~mm}$. Furthermore, the spatial resolution of our system was $63 \mu \mathrm{m}$ at the working distance of $50 \mathrm{~mm}$ [Fig. 4(c)] and $79 \mu \mathrm{m}$ at the working distance of $75 \mathrm{~mm}$ [Fig. 4(d)].

\subsection{System Sensitivity}

Figures 4(e)-4(f) show the sensitivity measurements of FFWT. The fused images of four EP tubes at the exposure time of $100 \mathrm{~ms}$ were shown in Fig. 4(e). Figure 4(f) plots the quantitative calculation of SBR $(13.8 \pm 0.3,8.3 \pm 0.9$, and $5.3 \pm 0.7 \mathrm{~dB})$ at the concentration of $1,0.1$, and $0.01 \mu \mathrm{M}$. The minimal detectable ICG concentration of this system was $0.01 \mu \mathrm{M}$ with the SBR of $5.3 \pm 0.7 \mathrm{~dB}$.

\subsection{In Vivo Feasibility Verification in Porcine Models}

Figure 5 shows the preclinical imaging of FFWT in six porcine models. To demonstrate the applications of the system for intraoperative lung cancer surgery guidance, the scenarios of thoracic duct visualization and intersegmental plane delineation were conducted. The representative color, NIR, and fused images of lung thoracic duct and pulmonary segments were shown in Fig. 5.

Figures 5(a) and 5(b) show the color and NIR images of the thoracic duct. The damage of thoracic duct leads to a serious complication (chylothorax) and reoperation of chylothorax patients. However, it is hard to distinguish the thoracic duct from surrounding tissues due to the thoracic duct with similar shape and color in comparison to surrounding tissue. ${ }^{29-31}$ With the NIR fluorescence imaging method, Fig. 5(c) shows the fused image that clearly indicated the location of the thoracic duct, which was difficult to distinguish from surrounding tissues in Fig. 5(a) only. This revealed that FFWT was feasible to visualize the thoracic duct and effectively avoid its unnecessary damage intraoperatively.

Meanwhile, pulmonary segmentectomy is a safe and minimally invasive technique in the lung cancer surgery. It has the potential to preserve lung function and reduce hospital costs, however, the identification of intersegmental plane requires the surgeons with a wealth of clinical experience and lacks a simple and effective technique to distinguish the targeted and nontargeted segments. ${ }^{26}$ Figures $5(\mathrm{~d})-5(\mathrm{f})$ are the color, NIR, and fused images of pulmonary segments collected by our dualchannel imaging system. Due to the lack of contrast between the ligation part and nonligation part, it is hard to identify pulmonary 




Fig. 5 Preclinical experiment results of the FFWT system. (a)-(f) Representative color, fluorescence and fused images of lung thoracic duct and intersegmental plane obtained by the system. (g) The average SBRs of the lung thoracic duct and intersegmental plane were $18.2 \pm 0.5$ and $21.5 \pm 0.3$, respectively. Scale bar: $2 \mathrm{~mm}$.

segments in the color image [Fig. 5(d)], whereas the boundary is easily seen in the fused image [Fig. 5(f)]. Furthermore, we use the SBR, which is defined as the ratio of the fluorescence signal in thoracic duct/ pulmonary segments to its surrounding tissues to evaluate NIR imaging effect and system performance. The quantitative measurements of the average SBR for lung thoracic duct and intersegmental plane in NIR images are $18.2 \pm 0.5$, and $21.5 \pm 0.3 \mathrm{~dB}$, respectively [Fig. 5(g)].

\subsection{In Vivo Near-Infrared Image-Guided Segmentectomy in Lung Cancer Patients}

In lung cancer surgery, segmentectomy is an important surgical option, but surgeons lack an efficient way to define intersegmental plane with the conventional white-light thoracoscopy. ${ }^{8}$ To address this clinical challenge, we performed NIR imaging guided segmentectomy using the FFWT system. Three patients with early-stage lung cancers were recruited in this preliminary study. As shown in Fig. 6, the high SBR $(19.6 \pm 0.8 \mathrm{~dB})$ of the fluorescent signal enabled easy and objective identification of the intersegmental plane, which revealed the potential of the clinical translation for using the system in MILCS.

\section{Discussion}

We have developed a color and NIR dual-channel thoracoscope system, namely FFWT, for real-time image-guided MIS. The system is capable of high-sensitivity NIR light collection, simultaneous optimized color and NIR dual-channel imaging, and ergonomic clinical application mainly based on the following four designs:



Fig. 6 Intraoperative imaging guided clinical segmentectomy using the FFWT system. The system provided surgeons a well-defined intersegmental plane with the SBR of $19.6 \pm 0.8 \mathrm{~dB}$ (Video 1, MOV, 913 KB [URL: http://dx.doi.org/10.1117/1.JBO.22.6.066002.1]). 
1. Optimal design of aperture size for maximizing the NIR light collection, ensuring sufficient depth of view, reducing stray light, and increasing brightness contrast of color imaging.

2. A self-developed compact optical path design, which applied the optical elements with transmission rates above $95 \%$, assembled the whole light splitter in a very small cylindrical chamber (internal diameter: $2 \mathrm{~cm}$ and internal length: $4 \mathrm{~cm}$ ) to minimize the light loss and system size.

3. A solution for achieving real-time and robust image registration, which designed two cameras with the same FoV and applied quick image registration algorithms.

4. The structure design, components size, and weight were optimized to satisfy real-time ergonomic clinical application and long-time handheld use.

Although a number of studies have already verified the significant clinical value of the NIR fluorescence image-guided MIS, most fluorescence thoracoscope systems are facing challenges of limited sensitivity and unpractical long exposure time. ${ }^{32,33}$ For normal clinical thoracoscopes, they have relatively small aperture and high signal loss for NIR-light transmission, so it is difficult to optimize the NIR fluorescence imaging in clinical MIS applications, where the high-sensitivity, high-contrast, and real-time imaging are highly needed. Currently, Liu et al. ${ }^{16}$ designed a single-channel endoscope system with a filter wheel to switch fluorescence and white-light imaging, and applied it for the highly specific detection of colonic adenomas. However, the single-channel imaging system had to acquire color and fluorescence images sequentially, which limited its usability in a clinical environment. Instead, Vivek et al. designed a dual-channel endoscopic imaging system to obtain white and NIR light information simultaneously. The system employed a prism-based two-CCD camera with both monochrome and color chips $1 / 3$ in. ${ }^{13}$ But the problem of this system was the limited sensitivity, which resulted in long exposure time for acquiring enough fluorescent signals. Different from Vivek, Glatz et al. ${ }^{14}$ used an electron-multiplying CCD camera with high NIR quantum efficiency to overcome the challenge of low fluorescent signal collection efficiencies. As a result, it indeed achieved highquality fused fluorescence and color imaging, but the overweight and oversize structure made it difficult to satisfy the clinical application requirements.

In this study, the dual-channel FFWT system was specially designed for ICG imaging in the real surgical conditions. A great amount of effort was carried out in the optical path design, imaging acquisition, illumination design, and the software development of the system, so that several critical challenges for the clinical translation of this optical imaging technology, ${ }^{29,34,35}$ such as the optimal NIR and white light simultaneous imaging, the minimization of the fluorescence signal loss, the minimization color and NIR images registration time with real-time exposure, as well as the reduction of the total size and weight for long-time handheld, were all effectively overcome or improved.

In addition, the feasibility of the FFWT system was evaluated through an in vivo preclinical porcine study and clinical lung cancer patient study. Although some researchers have applied the ICG-fluorescent imaging technique in segmentectomy, they used a single-camera fluorescence thoracoscope, which is inconvenient to acquire color and NIR images sequentially. ${ }^{23-25,36}$
Sekine et al. ${ }^{37}$ designed a color and NIR dual-channel imaging system applying for open segmentectomy. However, the dualchannel imaging system has not been tested in the thoracoscopic segmentectomy. The results indicated that FFWT not only identified the intersegmental plane with high SBR but also did not disturb the clinical flow by simultaneous color and NIR imaging guidance. However, since this study only recruited three lung cancer patients in the preliminary clinical trial, the performance of the FFWT system for imaging the thoracic duct and pulmonary segments needs to be further verified in a much larger population. The application of using the system and ICG for intraoperative imaging of lung cancer nodules is also needed to explore in the future.

In summary, the newly developed FFWT system can provide real-time NIR, color, and fused imaging simultaneously in MILCS. It demonstrated high sensitivity for ICG signal detection and offered high optical contrast for fast and objective identification of the thoracic duct and pulmonary segments intraoperatively in both preclinical porcine and clinical patient experiments. We believe that this advanced dual-channel optical imaging technology holds great promise for intraoperative guidance in clinical MILCS.

\section{Disclosures}

The authors declare no conflict of interest.

\section{Acknowledgments}

This work is supported by the National Basic Research Program of China (973 Program) under Grant No. 2015CB755500, the National Natural Science Foundation of China under Grant Nos. 81227901, 81527805, 81501594, 61671449, 61231004, 61501462, the Scientific Research and Equipment Development Project of Chinese Academy of Sciences under Grant No. YZ201359, and the Key Research Program of the Chinese Academy of Sciences under Grant No. KGZD-EW-T03. This pilot study was approved by Institutional Ethics Committee of Peking University People's Hospital (2015PHB157-01) and registered with the name of "Phase 1 study of NIR fluorescence guided thoracic surgery using ICG in ClinicalTrials.gov" (Grant No. NCT02611245).

\section{References}

1. R. L. Siegel, K. D. Miller, and A. Jemal, "Cancer statistics, 2016," CA Cancer J. Clin. 66(1), 7-30 (2016).

2. A. Jemal et al., "Cancer statistics, 2008," CA Cancer J. Clin. 58(2), 7196 (2008)

3. R. Wender et al., "American Cancer Society lung cancer screening guidelines," CA Cancer J. Clin. 63(2), 106-117 (2013).

4. C. F. Yang et al., "Long-term survival following open versus thoracoscopic lobectomy after preoperative chemotherapy for non-small cell lung cancer," Eur. J. Cardiothorac. Surg. 49(6), 1615-1623 (2016).

5. C. Chi et al., "Intraoperative imaging-guided cancer surgery: from current fluorescence molecular imaging methods to future multi-modality imaging technology," Theranostics 4(11), 1072-1084 (2014).

6. S. Keereweer et al., "Optical image-guided cancer surgery: challenges and limitations," Clin. Cancer Res. 19(14), 3745-3754 (2013).

7. Q. T. Nguyen and R. Y. Tsien, "Fluorescence-guided surgery with live molecular navigation-a new cutting edge," Nat. Rev. Cancer 13(9), 653-662 (2013)

8. J. Wright, "The eyes of the operation," Nature 502(7473) S88-S89 (2013).

9. A. L. Vahrmeijer et al., "Image-guided cancer surgery using near-infrared fluorescence," Nat. Rev. Clin. Oncol. 10(9), 507-518 (2013). 
10. N. Thekkek et al., "Quantitative evaluation of in vivo vital-dye fluorescence endoscopic imaging for the detection of Barrett's-associated neoplasia," J. Biomed. Opt. 20(5), 56002 (2015).

11. A. Matsui et al., "Real-time near-infrared fluorescence-guided identification of the ureters using methylene blue," Surgery 148(1), 78-86 (2010).

12. P. B. Garciaallende et al., "Towards clinically translatable NIR fluorescence molecular guidance for colonoscopy," Biomed. Opt. Express 5(1), 78-92 (2014).

13. V. Venugopal et al., "Design and characterization of an optimized simultaneous color and near-infrared fluorescence rigid endoscopic imaging system," J. Biomed. Opt. 18(12), 126018 (2013).

14. J. Glatz et al., "Concurrent video-rate color and near-infrared fluorescence laparoscopy," J. Biomed. Opt. 18(10), 101302 (2013).

15. G. Oh et al., "Intravital imaging of mouse colonic adenoma using MMPbased molecular probes with multi-channel fluorescence endoscopy," Biomed. Opt. Express 5(5), 1677-1689 (2014).

16. Z. Liu et al., "In vivo targeting of colonic dysplasia on fluorescence endoscopy with near-infrared octapeptide," Gut 62(3), 395-403 (2013).

17. Y. Park et al., "Spraying quantum dot conjugates in the colon of live animals enabled rapid and multiplex cancer diagnosis using endoscopy," ACS Nano 8(9), 8896-8910 (2014).

18. Y. Pan et al., "Endoscopic molecular imaging of human bladder cancer using a CD47 antibody," Sci. Transl. Med. 6(260), 260ra148 (2014).

19. S. G. Demos, M. C. Jacobson, and R. D. White, "In vivo testing of a prototype system providing simultaneous white light and near infrared autofluorescence image acquisition for detection of bladder cancer," $J$. Biomed. Opt. 17(3), 0360111 (2011).

20. Y. Ashitate et al., "Real-time simultaneous near-infrared fluorescence imaging of bile duct and arterial anatomy," J. Surg. Res. 176(1), 7-13 (2012).

21. Q. R. Tummers et al., "First experience on laparoscopic near-infrared fluorescence imaging of hepatic uveal melanoma metastases using indocyanine green," Surg. Innov. 22(1), 20-25 (2015).

22. M. J. Pucci et al., "Laparoscopic approaches to gastric gastrointestinal stromal tumors: an institutional review of 57 cases," Surg. Endosc. 26(12), 3509-3514 (2012).

23. N. Misaki et al., "New clinically applicable method for visualizing adjacent lung segments using an infrared thoracoscopy system," J. Thorac. Cardiovasc. Surg. 140(4), 752-756 (2010).

24. S. Tarumi et al., "Clinical trial of video-assisted thoracoscopic segmentectomy using infrared thoracoscopy with indocyanine green," Eur. J. Cardiothorac. Surg. 46(1), 112-115 (2014).

25. R. Waseda et al., "A novel fluorescence technique for identification of the pulmonary segments by using the photodynamic diagnosis endoscope system: an experimental study in ex vivo porcine lung," $J$. Thorac. Cardiovasc. Surg. 146(1), 222-227 (2013).

26. Y. Oh et al., "Intraoperative fluorescence image-guided pulmonary segmentectomy," J. Surg. Res. 199(2), 287-293 (2015).

27. H. Nomori, C. Yue, and H. Sugimura, "Systemic and regional pulmonary function after segmentectomy," J. Thorac. Cardiovasc. Surg. 152(3), 747-753 (2016).

28. P. Misthos, M. A. Kanakis, and A. G. Lioulias, "Chylothorax complicating thoracic surgery: conservative or early surgical management?," Updates Surg. 64(1), 5-11 (2012).

29. G. N. Box et al., "Comparative study of in vivo lymphatic sealing capability of the porcine thoracic duct using laparoscopic dissection devices," J. Urol. 181(1), 387-391 (2009).

30. A. S. Bryant et al., "The incidence and management of postoperative chylothorax after pulmonary resection and thoracic mediastinal lymph node dissection," Ann. Thorac. Surg. 98(1), 232-237 (2014).

31. M. Girotra et al., "Endoscopic ultrasound-guided transesophageal thoracic duct puncture in a swine model: a survival study," J. Laparoendosc. Adv. Surg. Tech. 23(7), 588-591 (2013).

32. T. Ali, P. L. Choyke, and H. Kobayashi, "Endoscopic molecular imaging of cancer," Future Oncol. 9(10), 1501-1513 (2013).

33. D. C. Gray et al., "Dual-mode laparoscopic fluorescence image-guided surgery using a single camera," Biomed. Opt. Express 3(8), 1880-1890 (2012).

34. C. Chi et al., "Use of indocyanine green for detecting the sentinel lymph node in breast cancer patients: from preclinical evaluation to clinical validation," PLoS One 8(12), e83927 (2013).
35. K. He et al., "Comparison between the indocyanine green fluorescence and blue dye methods for sentinel lymph node biopsy using novel fluorescence image-guided resection equipment in different types of hospitals," Transl. Res. 178, 74-80 (2016).

36. S. Iizuka et al., "Predictors of indocyanine green visualization during fluorescence imaging for segmental plane formation in thoracoscopic anatomical segmentectomy," J. Thorac. Dis. 8(5), 985-991 (2016).

37. Y. Sekine et al., "A simple and effective technique for identification of intersegmental planes by infrared thoracoscopy after transbronchial injection of indocyanine green," J. Thorac. Cardiovasc. Surg. 143(6), 1330-1335 (2012).

Yamin Mao is currently a postgraduate student with the Key Laboratory of Molecular Imaging of Chinese Academy of Sciences, Institute of Automation, Chinese Academy of Sciences, Beijing, China. She received her BE degree in automation engineering of Beijing Technology and Business University in 2013. Her main research interests include optical molecular imaging and fluorescence image-guided surgery.

Kun Wang is currently an associate professor with the Key Laboratory of Molecular Imaging of Chinese Academy of Sciences, Institute of Automation, Chinese Academy of Sciences, Beijing, China. He received his $\mathrm{PhD}$ degree in medical imaging of Newcastle University in 2011. His area of research encompasses the development of new methods and instrumentations for biological and medical imaging, especially in the field of optical and multimodality molecular imaging.

Kunshan $\mathrm{He}$ is currently a postgraduate student with the Key Laboratory of Molecular Imaging of Chinese Academy of Sciences, Institute of Automation, Chinese Academy of Sciences, Beijing, China. $\mathrm{He}$ received his $\mathrm{BE}$ degree in automation engineering of Chongqing University in 2014 . His main research interests include optical molecular imaging and wireless wearable fluorescence surgical navigation system study.

Jinzuo Ye is currently a postgraduate student with the Key Laboratory of Molecular Imaging of Chinese Academy of Sciences, Institute of Automation, Chinese Academy of Sciences, Beijing, China. He received his $B S$ degree in software engineering from Nankai University in 2011 and MS degree in computer technology from Institute of Automation, Chinese Academy of Sciences, in 2014. His main research interests include multimodality molecular imaging and fluorescence molecular tomography.

Fan Yang is a professor of thoracic surgery in the Department of Thoracic Surgery, Peking University People's Hospital. He received his MD degree in thoracic surgery from Peking University in 2001. From 2001 to 2004, he was a postdoctoral fellow in the Cancer Center of Tulane University. Since 2005, he has been a professor of Peking University People's Hospital. His current research interest is minimally invasive thoracic surgery.

Jian Zhou is currently an attending of thoracic surgery in the Department of Thoracic Surgery, Peking University People's Hospital. He received his BS degree in clinical medicine from Peking University Health Science Center in 2006 and his MD degree in thoracic surgery from Peking University in 2009. He was the leader of the research group of near-infrared guided thoracic surgery in his department.

Hao Li is currently a surgical resident of thoracic surgery in the Department of Thoracic Surgery, Peking University People's Hospital. He received his BS degree in clinical medicine from Peking University Health Science Center in 2014, and his MD degree in thoracic surgery from Peking University in 2017. His main research interests are nearinfrared guided thoracic surgery and liquid biopsy of lung cancer.

Xiuyuan Chen is currently a senior surgical resident of thoracic surgery in the Department of Thoracic Surgery, Peking University People's Hospital. He received his BS degree in clinical medicine from Peking University Health Science Center in 2011, and his MD degree in thoracic surgery from Peking University in 2014. His main research interests are applications of artificial intelligence and nearinfrared guided surgery in thoracic surgery. 
Jun Wang is a professor and director in the Department of Thoracic Surgery, Peking University People's Hospital. He received his master of medicine degree in thoracic surgery from Peking University in 1989. From 1997, he successively worked at the University of Washington, the University of Chicago, Harvard University, and Mayo Clinic. His current research interest is minimally invasive thoracic surgery. $\mathrm{He}$ has authored or coauthored more than 200 research papers in the international journals and conferences.

Chongwei Chi is currently an assistant professor with the Key Laboratory of Molecular Imaging of Chinese Academy of Sciences, Institute of Automation, Chinese Academy of Sciences, Beijing, China. He received his $\mathrm{BE}$ degree in automation engineering from
Jiangsu Normal University in 2008, his ME degree in control engineering from Dalian Jiaotong university in 2011 and his PhD degree in pattern recognition from Chinese Academy of Sciences. His main research interests are focused on biomedical imaging.

Jie Tian is a professor and director of the Key Laboratory of Molecular Imaging of Chinese Academy of Sciences, Institute of Automation, Chinese Academy of Sciences, Beijing, China. He received his PhD degree in artificial intelligence from Chinese Academy of Sciences in 1992. Since 1997, he has been a professor of Chinese Academy of Sciences. His current research interests include medical image process and analysis and pattern recognition. 